\title{
12. THE MOTIONS OF BODIES CLOSE TO COMMENSURABILITIES WITH JUPITER
}

\author{
A. T. SINCLAIR
}

Royal Greenwich Observatory, Herstmonceux Castle, Hailsham, Sussex, England

\begin{abstract}
The theory of the libration motion of a body close to a commensurability in mean motions with Jupiter is briefly described, and the importance of such motions in that they enable the body to avoid close approaches to Jupiter is discussed. By means of numerical integrations of the equations of motion some of the work that has been done on librations in orbits of low eccentricities is extended to higher eccentricities, so that the results can be applied to cometary orbits. By making some approximations the results are expressed in a very simplified form which makes it possible to compare the sizes of libration regions. This is done for six interior commensurabilities, namely the $3: 1,2: 1,5: 3,3: 2,7: 5$ and $4: 3$ commensurabilities.
\end{abstract}

